\title{
The role of symmetry in determining perceived centers within shapes
}

\author{
MARCO DAVI \\ University of Padua, Padua, Italy \\ and \\ M. ANNE THOMAS DOYLE and DENNIS R. PROFFITT \\ University of Virginia, Charlottesuille, Virginia
}

\begin{abstract}
This study was designed to assess the effects of symmetry and plane of presentation on the determination of the perceptual center of flat figures. Experiment 1 demonstrates the existence of effects in improving center determination, both in the number of sides of the shape and in rotational and reflective symmetry (confounded in the experiment). Experiment 2 shows that the presentation plane has no effect on center determination. In Experiment 3, we divide the effects of the two symmetry types, showing that rotational symmetry alone is as effective as the presence of both symmetry types-that is, the presence of symmetry axes is not very useful in finding perceived centers.
\end{abstract}

Research on the perception of flat-figure centers has shown a noticeable consistency as to where people see the center of flat figures (Davi, 1989; Gatti, 1924). In the present study, the effects of symmetry (reflective and rotational) and number of sides were assessed. To study the effects of these factors, it is necessary to know the relationship between the perceptual center and the mathematical centers and, in particular, which of the mathematical centers is the nearest to the perceptual center, so that the distance between these two can be used as a measure.

In every figure, there are four mathematical centers (Beghi, Vicario, \& Zanforlin, 1983): the geometric center, the barycenter of the vertices, the barycenter of the contour, and the barycenter of the area. The geometric center is defined as the locus of equal distances from the vertices. However, not all figures have a proper geometric center, in which case, an approximation of the geometric center (called centroid) can be calculated, defined as that point almost equidistant from the vertices. This definition is an extension of that of the geometric center, and it ensures that such a point exists for all convex shapes.

As for the three barycenters, it is possible to describe them in terms of what is taken into account in the computation. If just the vertices of a figure are considered (giving an equal weight to each of them), what will be found will be the barycenter of the vertices. If the whole fig-

This paper was written as partial fulfillment of $M$. Davi's PhD thesis. The research was supported by Air Force Office of Scientific Research Grant AFOSR-91-0057 and NASA Grant NCA2-468 to D. Proffitt. We thank Steve Jacquot for his assistance with the computer programs. Address correspondence to Dennis R. Proffitt, Department of Psychology, Gilmer Hall, University of Virginia, Chalottesville, VA 22903-2477. ure's contour is considered instead (giving the same weight to each of its points), computing the barycenter will provide the contour barycenter. Finally, if the whole figure is considered (giving the same weight to each point on the contour and within it), computing the barycenter will give the barycenter of the area (for an example of these four mathematical centers placed within a shape, see Figure 1). These four different centers sometimes coincide (e.g., in regular figures).

Davi (1989) assessed which mathematical center is nearest to the perceptual one and found the perceptual center to be a deviation from the barycenter of the vertices. This result was obtained using both contour drawings (isosceles triangles) and solid color figures (three shapes, one of which is depicted in Figure 1).

In another work, Proffitt, Thomas, and O'Brien (1983) found that subjects rely on the information given by the contour. In that study, there were shapes with uniform luminance distribution and shapes with luminance distribution that varied across the shape. Luminance variation, however, causes no change in the placement of the perceptual center, so it can be stated that the perceived center was not that of the luminance distribution, but rather a center whose finding was based on the information given by the shape's contour (and not by the borders of the stripes).

The aforementioned study has to be briefly summarized, because it is the only one that has been concerned with the effect of symmetry on center perception, and because it provides the most powerful suggestion of an effect of symmetry in this perceptual task. Proffitt et al. (1983) used figures with different luminance distribution, stationary or rotating. In four of their five shapes, all the mathematical centers coincided (because they were regular figures), and in the fifth figure the utilized mathematical 


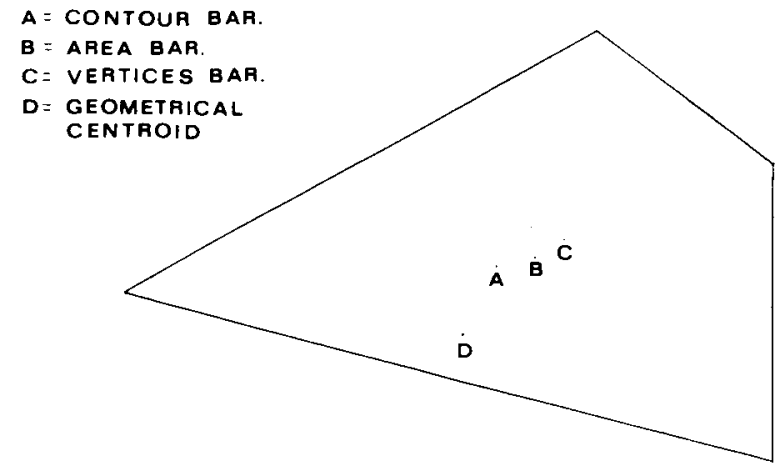

Figure 1. A shape (used in an experiment by Beghi et al., 1983) and its four mathematical centers.

center was the area barycenter. Luminance manipulation had no effect on perceptual center localization: in every case, it was nearer to the mathematical centers of the shape than to the luminance distribution center. Their five figures also varied with respect to order of symmetry: circle, infinite; square, four; equilateral triangle, three; oval, two; amoeboid figure, one. The variability of judgments increased inversely to the order of symmetry.

With respect to the Proffitt et al. (1983) study, some observations can be made. First, there was a "hole" in the order of symmetry progression, from four to infinite. It would be interesting to verify if the observed effect was also present in a series of figures with orders of symmetry greater than four, but not infinite. (Gatti, 1924, already noted that for circles the error is null.) Also, most of the utilized figures were regular. This means that, together with the increase in order of symmetry, there was a parallel increase in the number of sides in the figure; the effect of the two factors was therefore confounded. All these problems were addressed in the present study.

Symmetry has proved to be an influential factor in many perceptual contexts. It is related to judged figural "goodness" (Garner \& Clement, 1963). Moreover, there is evidence that it can play an important role in pattern recognition (Hartley, 1982; Schulze, Baurichter, Gerling, \& Grobe, 1977). Finally, eye-movement studies have dem- onstrated the existence of an interaction between symmetry and complexity (Locher \& Nodine, 1973). As for center perception, Proffitt et al.'s (1983) results provide the strongest motivation for looking for an effect of symmetry. We expect that the presence of reflective symmetry would reduce the systematic error (the distance between the perceptual center and a mathematical center), because this symmetry implies redundant information (i.e., one half of the figure is the mirror image of the other) and intrinsic axes for the figure are given. A differential effect for the two symmetry types could also be present, because when both symmetries are present the reflectional axes (which intersect at the center) could give valuable aid in the process of finding the center (see Figure 2A), whereas when only rotational symmetry is present, no such axes are available. However, rotational symmetry could have an effect, because the sides of the figure are not all of different lengths, thus providing a certain regularity (see Figure 2B). Indeed, the number of sides could, by itself, also have an effect on center determination. In fact, if we remember that the mathematical center nearest to the perceptual center is the barycenter of the vertices, then increasing the number of sides will give more vertices, and hence more information available for finding the center, which could result in a decrease of the systematical error. Furthermore, the perceptual "drawing" of lines from the vertices or from the midpoints of sides was the first hypothesis about how the perceptual center is found (Gatti, 1924).

Three factors were examined in Experiment 1: symmetry, number of sides of the shape, and moving versus static shapes. Four different sets of shapes, of increasing symmetry, were used. Reflective and rotational symmetry were confounded for all except Set 1, which had reflective symmetry only. Experiment 2 tested whether a difference in presentation plane (vertical vs. horizontal) would influence the results. In Experiment 3, the effects of the two symmetry types were disentangled (there were three kinds of shapes: with both rotational and reflectional symmetries, with just rotational symmetry, and with no symmetry). The other factors were the number of sides, and concave versus convex shapes.

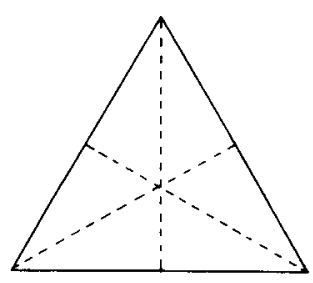

A

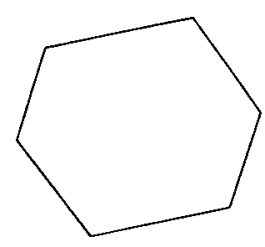

三

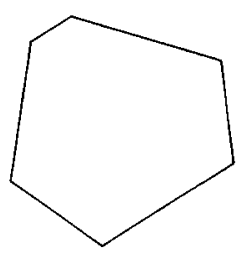

c

Figure 2. (A) An equilateral triangle with its three axes of reflective symmetry intersecting at the center. Equilateral triangles have both symmetry types. (B) A shape that has rotational symmetry but no reflective symmetry. However, the sides of the shape consist of pairs of equal length, and this provides a certain regularity, making it easier to find the center than is possible with a shape such as that depicted in (C), which has no symmetry of any kind. 
The main purpose in Experiment 1 was to establish whether symmetry had an effect on center determination. As mentioned above, the best explanation for this comes from Proffitt et al.'s (1983) study. However, in that experiment, there was only one shape per symmetry set, so the authors could only speculate about the role of symmetry in center determination. In the present study, there were four shapes per symmetry set, so it was possible to generalize from configural effects to symmetry effects.

\section{EXPERIMENT 1}

In this experiment, subjects viewed a variety of moving and static shapes, and were asked to find the center of each shape.

We formulated four hypotheses, as follows: (1) As the degree of symmetry (both rotational and reflective) increases for a set of shapes, observers would be more accurate in determining the center of each shape; (2) as motion provides additional information in tasks involving center determination, the centers of shapes would be determined more accurately when the shapes are rotating and not static; (3) for the task of center determination, deviation of responses would be along the axis of symmetry rather than off the symmetry axis for shapes with one axis of reflective symmetry; and (4) subjects would be more accurate at determining the center of shapes with many sides than shapes with few sides, averaged across symmetry.

\section{Method \\ Subjects \\ Twenty University of Virginia students participated to fulfill part of a course requirement in introductory psychology. Ten subjects served in Condition 1 , and 10 served in Condition 2 . The subjects were tested individually.}

\section{Stimuli and Apparatus}

The apparatus had three major components: the revolving $x-y$ plotter, a camera, and the monitor viewed by the subject.

The revolving $x-y$ plotter (described in detail by Proffitt $\&$ Simmons, 1980) allowed the subject to affect the location of a stimulus mounted on an unseen disk. The subject viewed a CRT screen with a very low brightness setting and a relatively high level of contrast. This, along with the lighting, ensured that the workings of the $x-y$ plotter were invisible to the subject, who could see only the shape as it appeared on a black background.

The control panel, placed on a tabletop between the subject and the video monitor, consisted of two pairs of buttons-one to move the shape back and forth along the $x$-axis, and the other to move it back and forth along the $y$-axis.

At the beginning of each session, a small dot was affixed near the center of the monitor screen which corresponded to the center of the plotter. This was done by positioning the glide in the center of the $x-y$ plotter and mounting a shape with a black dot at its center to the joiner pin. The small dot on the monitor screen was adjusted for each subject so that it coincided exactly with the center of the plotter. The individual adjustment of the dot on the monitor screen was necessary to control possible parallax errors due to each subject's unique head position and posture. It has been demonstrated (Proffitt et al., 1983) that the dot on the monitor screen does not affect the observers' responses.
The stimuli were the 16 shapes depicted in Figure 3. They varied from one to four axes of reflective symmetry, and from one to fourfold rotational symmetry. For all the shapes, these two symmetry types covaried together. Therefore, all shapes within a particular symmetry group had the same amount of reflective and rotational symmetry.

There were four examples of each symmetry group. Each of these "loosely" belonged to categories associated with a different number of side's, which is illustrated by the horizontal groupings of shapes in Figure 3. Within each group, the shapes' number-of-sides level was held relatively constant (see the number in the upper right corner of each shape cell).

All 16 shapes had disk size in common. Disk size refers to a particular characteristic of rotating solid shapes, and corresponds to the largest circle that can be drawn inside a shape's boundaries. The area of a shape around the center that has no indentations or protuberances seems to "hang together" as a disk when the shape rotates at, or nearly around, the center. This disk varies in size independently of surface area; it depends, rather, upon how close to the center the indentations come. The disk was held at a constant size of $42 \mathrm{~mm}$ (visual angle of less than $5^{\circ}$ ) for all 16 shapes as they appeared on the monitor screen.

The stimuli were made from heavy white posterboard and were mounted on black disks that could be attached one at a time to the $x-y$ plotter at the joiner pin. The orientation of the mounted shapes was held constant for all presentations (they were the same as those depicted in Figure 3) so that the coordinate values reported were relative to the centers at the origin. The specific dimensions of the shape (in millimeters) along the $x$ - and $y$-axes are given in Figure 3 . There was some variability in the dimensions of the shapes with respect to length and width. The visual angle ranged from $5^{\circ}$ to

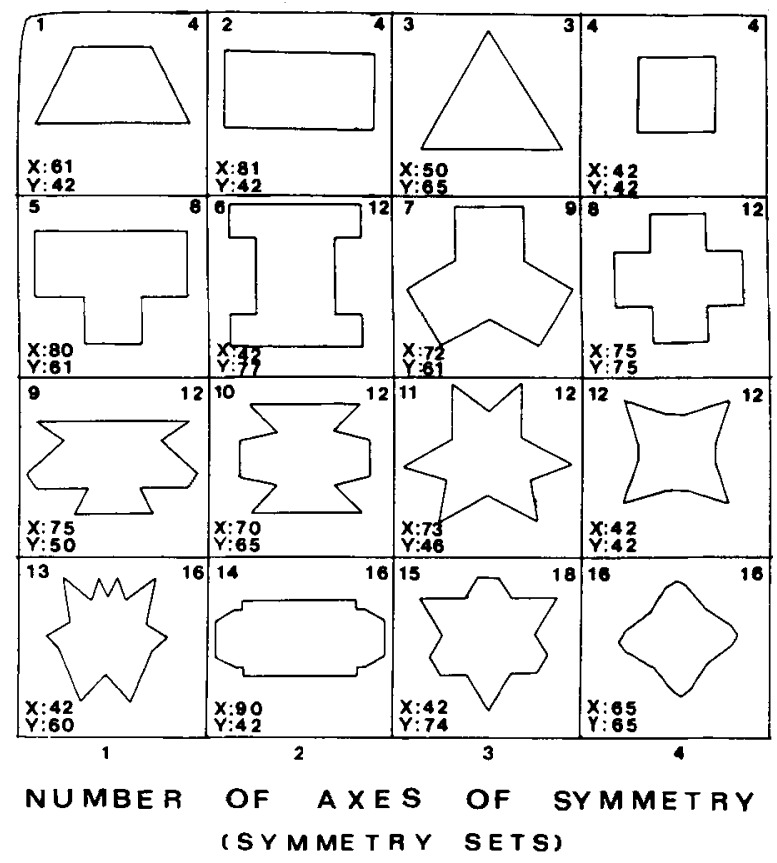

Figure 3. Stimuli of Experiment 1. All shapes are shown at the orientation used for all static presentations. The values for the sizes of the shapes along the $x$-and $y$-axes are given in millimeters as $x$ : and $y$ :. The number used to identify each shape appears in the upper left corner of each cell, and the number in the upper right corner indicates the number of vertices in that shape. 
$2^{\circ}$ for the major axis of the shapes. No shape had a visual angle along the minimum dimension of less than $2^{\circ}$.

The stimuli were generated from the class representing the prototypical shapes (Figure 3; shapes 1, 2, 3,4). Variations were made on this class of figures so that across the symmetry dimension, number of sides was as constant as possible.

\section{Procedure}

Condition 1. The subjects were tested during two $1-\mathrm{h}$ sessions. Each was seated in front of the monitor screen with the control panel within easy reach. The general procedure was described and training commenced. This involved familiarizing the subject with the control panel so that he/she became adept at directing the motion of the shape on the monitor screen. During this period, the subject was shown a rotating white octagon (the major axis equaled $100 \mathrm{~mm}$ ) that was similar to the test stimuli. The center of the shape was initially placed at some distance from the origin of the plotter. The subject was then directed to "find the point where the shape just sits and spins, no longer moving as a whole on the screen." While using this shape, the subject was given assistance and direction by the experimenter so as to facilitate manipulation of the buttons and, consequently, of the shape. After a short period, each subject was required to demonstrate competency with the technique by adjusting the octagon to within $1 \mathrm{~mm}$ of the center on both the $x$ - or $y$ axis on three successive trials. At this point, the subject was informed that he/she would be seeing a number of other shapes on the screen and that the task would be the same for each. The familiarization procedure lasted approximately $15-20 \mathrm{~min}$.

During the test trials, 8 of the 16 shapes were presented in random order during the first session, and the remainder were presented during the second. After each trial, the experimenter measured the displacement of the perceived center from the origin of the $x-y$ plotter.

For the subjects' second session, the procedure was repeated with a condensed explanation of the function and operation of the apparatus. Proficiency was demonstrated by again reaching the same criterion.

Condition 2. The procedure was similar to that of Condition 1, except that the shapes were not revolving. Initially, the shape was seen displaced some distance from the dot previously affixed to the monitor screen (coinciding with the origin or center of the disk of the plotter). The shapes were aligned for presentation, as depicted in Figure 3. The subject was asked to move the shape by pressing the two pairs of buttons so that "the center of the shape falls directly under the dot on the screen." One pair of buttons caused the shape to move in a $125^{\circ}$ or $305^{\circ}$ direction, while the other pair caused movement in a $35^{\circ}$ or $215^{\circ}$ direction. Because there was no rotational component of motion, the shape moved only while the buttons were pressed.

During this procedure, the criterion level was the same as that used in the moving condition (to come within $1 \mathrm{~mm}$ on the $x$ - or $y$-axis, using the octagon on three successive trials). After reaching the criterion, however, participants in Condition 2 saw the 16 stimuli presented in a random order during a single session lasting approximately $1 \mathrm{~h}$.

\section{Results}

The measurements taken were the values on the $x$ - and $y$-axes for the perceived centers of each shape. These provided scores that could be used to determine the mean location of the perceived centers for the individual shapes. A measure of variability was computed by using the $x$ and $y$ values to determine a distance measure $(d)$ of the perceived center from the actual center for each shape. This distance measure allowed the variability of responses for the shapes to be explored. A multivariate analysis of variance (ANOVA) was used to analyze the resultant measures. The Pillai-Bartlett criterion with appropriate conversion to an approximate $F$ statistic was employed.

By averaging over the two motion conditions, a test of the symmetry effect for the measure $d$ was performed and found to be significant $[F(1,18)=3.768, p=.032]$. The means for each of the four symmetry sets, regardless of the motion conditions, suggested that, in fact, the distance, $d$, was inversely related to the degree of symmetry. In other words, as the shapes' symmetry properties increased, the observers' responses deviated less from the actual center of the shape (Hypothesis 1 was confirmed).

By ignoring differences in the symmetry properties and looking across all shapes, a condition effect can be seen to exist for the $d$ measure $[F(1,18)=14.225, p=.001]$. However, it was not in the predicted direction-for Condition 1 , the mean $d$ (rotational) was $3.185 \mathrm{~mm}(S D=$ 1.18), and for Condition 2, (static) it was $1.727 \mathrm{~mm}$ $(S D=.3375)$ (see Figure 4). There was less variability in subjects' responses when the shapes were not moving (there is an effect of motion, but it is opposite to $\mathrm{Hy}$ pothesis 2).

Two questions can be raised. First, do the perceived centers coincide with the configural centers? Comparison of the perceived and configural centers can be made by determining if the mean of the subjects' responses is different from zero. In the condition with rotation, the

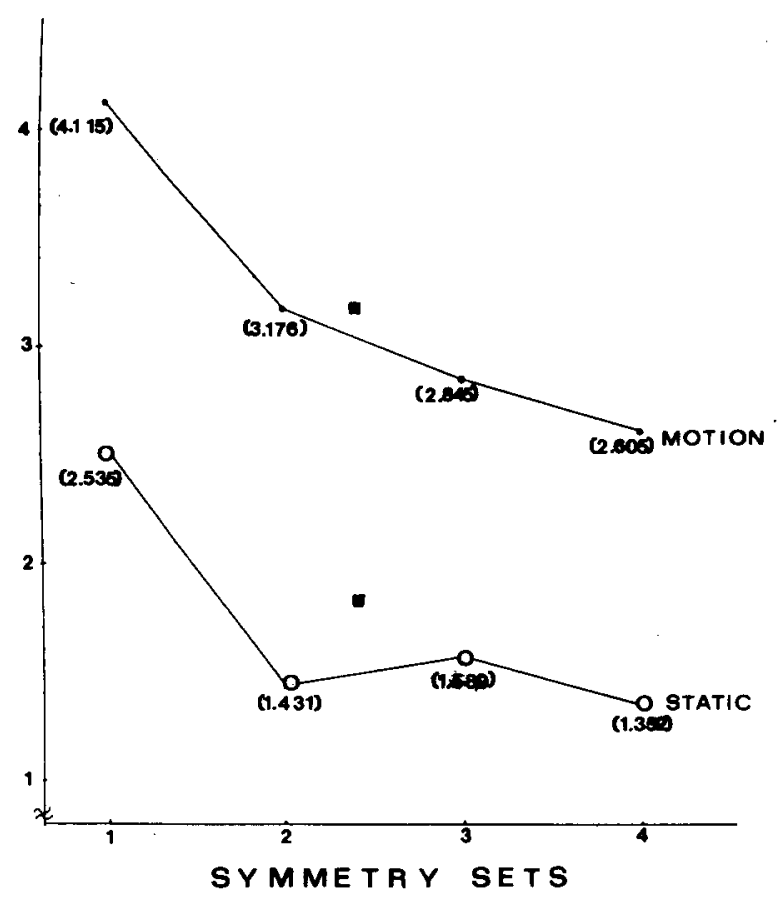

Figure 4. Means of the distance $d$ reported by the symmetry sets within each condition (the black squares indicate the means for each condition). The symmetry effect was significant at $p=.032$, and the motion effect was significant at $p=.001$; the interaction was not significant. 
perceived and configural centers did not coincide on the $y$-axis $[F(1,9)=14.12, p=.004]$. In other words, the perceived center was significantly different from zero. However, the mean of $y$ was $.8512 \mathrm{~mm}-$ not a great displacement. There was no displacement of perceived and configural centers on the $x$-axis, nor were there differences on either axis in the static condition.

Second, within each condition, are the responses for the symmetry sets different from each other? In the moving condition, the failure of $d$ to be significant suggests that the variability of subjects' responses did not differ between the symmetry sets. In the static condition (Condition 2), significant differences exist between the symmetry sets on $d[F(1,9)=13.439, p=.003]$. However, when a series of pairwise comparisons were made, Symmetry Set 1 was found to differ from the other three sets. No other differences between pairs were found. This suggests that Set 1 was responsible for the symmetry effect in the static condition. Measurements were taken from the area barycenter. This barycenter coincided with the other ones in all shapes, except those of Symmetry Set 1. However, the distance between the three barycenters (vertices barycenter, contour barycenter, and area barycenter) for shapes of Symmetry Set 1 was, on average, only $.25 \mathrm{~cm}$, whereas the effect averaged $1.02 \mathrm{~cm}$.

Because the literature regarding orientation suggests that observers are most sensitive to symmetry axes aligned with the vertical (Humphreys, 1983; Palmer, 1985; Palmer \& Hemenway, 1978; Rock, 1973; Rock \& Leaman, 1963), we expected that, in the static condition, the perceived centers of shapes would tend to be close to zero on the $x$-axis and show greater variability around the $y$ axis (the shapes were aligned for presentation as depicted in Figure 3). A review of the mean values along the $y$ axis, $y$, supported this prediction. For the shapes of Symmetry Set 1, $y=-.612(S D=1.161)$; of Set 2, $y=.388(S D=1.028) ;$ of Set $3, y=-.588$ $(S D=.866) ;$ and of Set $4, y=-.562(S D=.744)$. These results do not indicate any strong differences for $y$ between the symmetry sets. However, a look at the standard deviations suggests that the variance of responses tended to decrease as the symmetry properties for the shapes increased. The subjects were very good at locating the vertical axis of symmetry, as indicated by the very small scores on the $x$-axis. For the shapes of Symmetry Set 1, $x=-1.025(S D=.984)$; of Set 2, $x=.125$ $(S D=1.143)$; of Set 3, $x=-.187(S D=.607)$; of Set $4, x=-.175(S D=.836)$ (Hypothesis 3 was confirmed).

Collapsing the data across conditions, significant differences were found between the number-of-sides levels on $d[F(3,16)=4.1685, p=.0232]$. The means for $d$ from the group of shapes with the lowest number of sides to the group with the highest number of sides were 3.111, $2.435,2.264$, and 2.014, respectively (Hypothesis 4 was confirmed).

\section{Discussion}

The results indicated a significant symmetry effect, particularly in the static condition. As stated earlier, Symmetry Set 1 was primarily responsible for this effect. The shapes in Set 1 share the characteristic of one common axis of reflective symmetry. This means that the shapes are bilaterally symmetrical, that is, the visual information around the symmetry axis is redundant to the subject. The other symmetry sets had 2, 3, or 4 axes of reflective symmetry. The information around each of these axes is (by definition) redundant. It is possible that this redundancy is the significant factor that "lumps together" the sets with the higher order symmetrical properties.

The literature suggests that observers are most sensitive to vertically oriented axes of symmetry, then to horizontal axes (Corballis \& Roldan, 1975; Olson \& Attneave, 1970; Palmer \& Hemenway, 1978; Rock, 1973) and, finally, least sensitive to diagonally oriented axes of symmetry (Rock \& Leaman, 1963). Figure 3 shows the symmetry axes for each of the shapes used. Notice that all of the sets have a vertical axis, Sets 2 and 4 also have horizontal axes, and Sets 3 and 4 have additional diagonal axes. It seems that the addition of either horizontal or diagonal axes provides the subject with enough added information to be more accurate in determining the centers of shapes.

A probable explanation for this has to do with the intrinsically different information available from shapes with one axis of symmetry from shapes with multiple symmetry axes. In the shapes of Symmetry Set 1, there is a single vertical axis of symmetry. To determine the center of these shapes, a subject has information regarding the placement of the axis from the redundancy on each side of the axis. However, the determination of the shape's center requires the location of a unique point along that axis-the $y$-axis in this study. For the shapes of Symmetry Sets 2, 3, and 4 , there are additional axes of symmetry that intersect at the shape's center. A subject can use the information available from the redundancy resulting from these imaginary lines. This means that a subject needs only determine that two (or more) axes of symmetry exist and "trace" them to their intersection to locate the center of the shape. Shapes with one axis of symmetry could make the subject more easily "miss the point."

The results lend support to this speculation. More variance of responses tended to occur along the $y$-axis for the shapes with only one axis of symmetry than they did for other shapes. This suggests that it was, in fact, more difficult to pinpoint the center for these shapes $(1,5,9$, and 13; Figure 3) than for other shapes that had other symmetry axes intersecting at the center.

The direction of the motion effect was surprising, but not totally unexpected. Proffitt et al. (1983) conjectured that it was most likely that as the shapes revolved, some configural information would be lost but be made up by the additional motion information. This speculation seems 
likely in the present study as well. The configural symmetry information may not have been as noticeable to subjects seeing rotating shapes. Rotating shapes were used to see if the minimization of relative motion could be employed by the subjects to find the center. Because performance was poorer in the moving condition, there was no evidence for or against the existence of a facilitatory effect from motion. ${ }^{1}$ On the other hand, there clearly must have been a loss of figural information; that is, with rotating shapes, less figural information seems to be available to the subjects. This is probably due to the fact that the detection of symmetry has orientational specificity. In other words, vertical symmetry is most salient, as cited above. Thus, the salience of symmetry is degraded by rotation, probably because when shapes are rotating, every existing symmetry axis is vertical only for a very short moment during each rotation. This may explain why there was a greater error for moving shapes.

An effect of number of sides was also observed: an increase in number of sides improves center finding. It may simply be that a higher number of sides, especially in a symmetrical shape, provides more redundant information that can be used to find the center. Or it may be that the side has a more fundamental role, possibly that of a real "informational unit" for finding the perceptual center. However, this cannot be decided on the basis of this experiment alone, because it is necessary to observe the effect of number of sides by itself, that is, in shapes that have no symmetry and with a different number of sides. This was done in Experiment 3.

The results of the above study provided the basis for designing Experiments 2 and 3. For all of the shapes except Set 1 , rotational and reflective symmetry were confounded. The symmetries might not affect the observer in the same way. The best evidence for this was shown in the difference found here between Set 1 and Sets 2, 3 , and 4 . While the shapes of Symmetry Set 1 all had one axis of reflective symmetry, it is also true that shapes having no axis of reflective symmetry have rotational symmetry. It would be interesting to see if the perceived center and the actual center for shapes without an axis of reflective symmetry but with rotational symmetry would be displaced more than they would for the shapes of Symmetry Set 1 or be about the same. It would also be interesting to make a similar comparison with shapes without any symmetry. The latter case would be particularly noteworthy, because the explanation suggested here for the process of the determination of the perceptual center (the symmetry axes intersection) cannot, by definition, apply to these shapes. In these shapes, it would also be possible to observe, by itself, the effect of the increase in number of sides. First, however, we would need to check to see if there was a difference due to the presentation plane.

\section{EXPERIMENT 2}

This experiment was designed to test whether there was a difference in locating perceptual centers due to the pre- sentation plane (frontoparallel vs. horizontal). Furthermore, we wanted to recheck to see if, in regular polygons, the systematical error (the distance between the perceptual center and the mathematical centers) would decrease as order of symmetry increased (making this factor vary on a wide range of values, without reaching the infinite).

\section{Method}

Subjects

Fourteen psychology students from the University of Padua served as subjects.

\section{Stimuli}

The stimuli were five regular geometrical figures, of increasing order of symmetry (that for regular figures is equal to the number of sides), drawn in black india ink ( $0.4 \mathrm{~mm}$ thick) on white paper $(21 \times 21 \mathrm{~cm})$. The extent of the major axis (in millimeters) for each of the figures employed was as follows: equilateral triangle, 46; square, 36; hexagon, 55; octagon, 53; and decagon, 62.

\section{Procedure}

The subject was seated in front of a table and was asked to quickly mark, with a sharpened pencil, a point at the center of each figure to be shown, one by one, at a distance of about $50 \mathrm{~cm}$. The drawings were presented twice to each subject: once on the table (horizontal plane) and once on a vertical reading deck (frontoparallel plane). To half of the subjects, the stimuli were presented first on the table, and to the other half they were first on the reading deck. In each condition, the stimuli were presented in random order.

\section{Results}

Measurements were made using a millimetric grid. The abscissa and ordinate values of the centers marked by the subjects were converted into distances from the mathematical centers (which, in these regular figures, are all coinciding) and standardized using Proffitt and O'Brien's (1982) procedure. This standardization makes it possible to compare distances obtained in figures differing in shape and size. These standardized distances were submitted to a two-way $(5 \times 2)$ repeated measures ANOVA. The factors were order of symmetry and presentation plane.

The main effect of order of symmetry was significant $[F(4,52)=70.719, p<.001]$, whereas the effect of presentation plane was not. The interaction was also not significant. The magnitude of the systematical error decreased as order of symmetry increased. In fact, for order of symmetry $3,4,6,8$, and 10 , the mean standardized distances $d$ of the perceptual center from the mathematical center were, respectively, $3.381, .671, .555, .628$, and .683 . However, on a pairwise comparison only, the first shape (triangle; order of symmetry 3 ) significantly differed from all the others [at least $F(1,6)=35.84, p<$ $.001]$. No other difference was found.

\section{Discussion}

The fact that the effect of presentation plane was not significant is in agreement both with Beghi et al.'s (1983) informal observations and with the ample literature on shape constancy phenomena. This result is also interpreta- 
ble in terms of Davi's (1989) hypothesis, which states that, in determining the center, the figure acts as the reference frame of itself. In other words, "up" and "down" are defined with respect to the figure.

The significance of order of symmetry confirms Proffitt et al.'s (1983) results. Together, these studies lead one to suppose that, in the process of determining the perceptual center, the information carried by symmetry is involved. Given that the presentation plane had no effect on center determination, Experiment 3 was conducted in the horizontal plane, which provided a more natural situation for the subjects, who had to mark the center with a pencil.

\section{EXPERIMENT 3}

In Experiment 3, our aim was to separate the effects of the two symmetry types, as well as the effect of the number of sides from those of symmetries. For this, we used three kinds of stimuli: one with both reflective and rotational symmetries, one with only rotational symmetry, and one with no symmetry. There were two series for each of these types-one composed of concave shapes and one composed of convex shapes. Since presentation plane does not influence center estimation (Experiment 2), all the stimuli were presented on a horizontal plane.

\section{Method}

Subjects

Twelve psychology students from the University of Virginia (different from those in Experiment 1) served as subjects.
Stimuli

All stimuli were drawn in black india ink ( $0.4 \mathrm{~mm}$ thick) on white paper $(21 \times 21 \mathrm{~cm})$.

Starting with three prototypes (an equilateral triangle, a square, and a pentangle), two sets of shapes were constructed by doubling the number of sides in the prototype: one of concave shapes and one of convex shapes (see Figure 5). Within each set, there were three series of shapes: one with both reflective and rotational symmetries (all the sides were of equal length), one with only rotational symmetry (the sides were of two lengths, alternated), and one with no symmetry (all the sides were of different lengths).

The extents of the major axis (in millimeters) of each of the prototypes were: triangle, 70; square, 56; and pentangle, 75. Across the shapes derived from a single prototype, the areas taken off from the prototype's shape were approximately equal (identical for concave shapes), as was the "compactness" of the shapes (evaluated by means of the second moments, or variances, of the shape; see Zusne, 1965, 1970).

\section{Procedure}

The procedure was essentially the same as in Experiment 2. However, in this experiment, all the stimuli were presented on the table (horizontal plane). To compensate up/down and left/right spatial biases, the stimuli were presented in random orientation. In all, 21 sheets were presented (the 18 experimental stimuli and the 3 prototypes), one by one, in random order.

\section{Results}

Measurements were made using a millimetric grid. The abscissa and ordinate values of the centers marked by the subjects were converted into distances from the vertices' barycenter (however, in 17 of the 21 shapes of the present experiment, all the mathematical centers coincided). The choice of this mathematical center as a reference point

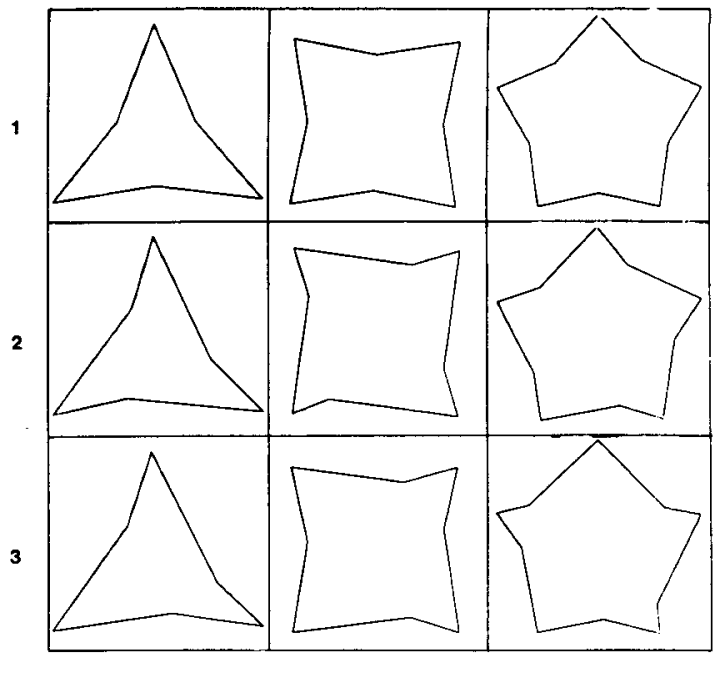

A

B

c

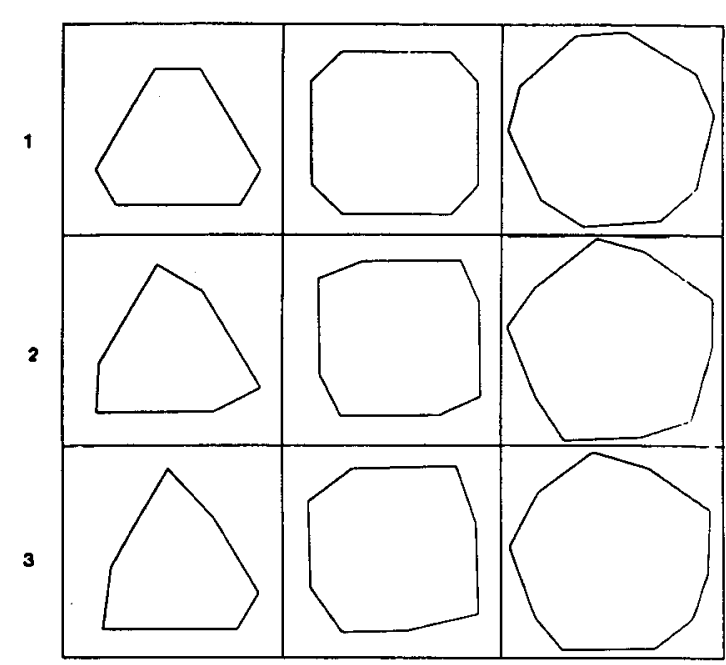

A
B

Figure 5. Experimental stimuli of Experiment 3. The left panel depicts the concave shapes, and the right panel shows the convex shapes. Within each panel, the first row consists of shapes with both kinds of symmetry; the second row consists of shapes with only rotational symmetry; and the third row consists of shapes with no symmetry. Shapes in column $A$ were made by doubling the sides of an equilateral triangle, those in column $\mathrm{B}$ by doubling the sides of a square, and those in column $\mathrm{C}$ by doubling the sides of a pentangle. 
is due to the fact that, in previous studies (Davi, 1989), it was found to be nearest to the perceptual center. Moreover, as in Experiment 1, in the 4 shapes with noncoinciding barycenters, distances between barycenters were very small. Here, we computed which barycenter was nearest to the mean perceptual center. In these four shapes, it proved to be the vertices barycenter. Distances were then standardized using Proffitt and O'Brien's (1982) procedure. The mean standardized distance for each experimental stimulus is given in Table 1.

The standardized distances were submitted to two repeated measures ANOVAs. The first one-way analysis (with three levels) was conducted on the prototypes. The second three-way analysis $(2 \times 2 \times 3)$ was conducted on the experimental stimuli. The factors were: concave versus convex shapes, symmetry type, and number of sides.

The analysis on the prototypes was not significant. In other words, the distance of error was about the same for every prototype.

In the analysis on the experimental stimuli, the following main effects and interactions were significant: concave versus convex shapes $[F(1,11)=8.521, p=.014]$, symmetry type $[F(2,22)=20.55, p<.001]$, concave versus convex $\times$ number of sides $[F(2,22)=5.221, p=$ $.014]$, and concave versus convex $\times$ symmetry type $\times$ number of sides $[F(4,44)=8.273, p<.001]$.

The means for concave and convex shapes were, respectively, 1.816 and 1.623 . This shows that subjects are more accurate in locating the centers of convex shapes than those of concave shapes. The means for symmetry type, when both symmetries, only rotational symmetry, or no symmetry were present were, respectively, 1.566, 1.403, and 2.189. This pattern of values is reproduced for the shapes obtained from each of the three prototypes, as can be seen in Table 2. On a pairwise comparison made by using the Newman-Keuls test, it was found that the nosymmetry level was significantly different from both the symmetry and the rotational symmetry levels $(p<.01$, in both cases). However, there was no difference between the symmetry and rotational symmetry levels. These results show that symmetry has a role in facilitating center determination, given that the distances between the actual and the perceived centers are much higher when no

Table 1

Average Standardized Distance for Each Experimental Stimulus

\begin{tabular}{lccc}
\hline & \multicolumn{3}{c}{ Prototype } \\
\cline { 2 - 3 } \multicolumn{1}{c}{ Symmetry Sets } & Triangle & Square & Pentangle \\
\hline & Concave & & \\
Both symmetries & 1.746 & 1.270 & 1.682 \\
Rotational symmetry & 1.489 & 1.491 & 1.447 \\
No symmetry & 2.272 & 1.670 & 3.279 \\
& Convex & & \\
Both symmetries & 1.578 & 1.627 & 1.317 \\
Rotational symmetry & 1.729 & .848 & 1.414 \\
No symmetry & 1.880 & 2.442 & 1.588 \\
\hline
\end{tabular}

Table 2

Mean Values for the Three Symmetry Sets for the Shapes Obtained from the Three Prototypes

\begin{tabular}{lccc}
\hline & \multicolumn{3}{c}{ Prototype } \\
\cline { 2 - 4 } \multicolumn{1}{c}{ Symmetry Sets } & Triangle & Square & Pentangle \\
\hline Both symmetries & 1.752 & 1.448 & 1.449 \\
Rotational symmetry & 1.609 & 1.170 & 1.430 \\
No symmetry & 2.076 & 2.056 & 2.434 \\
\hline
\end{tabular}

Table 3

Mean Values for Concave or Convex Shapes, Obtained From the Three Prototypes

\begin{tabular}{lccc}
\hline & \multicolumn{3}{c}{ Prototype } \\
\cline { 2 - 4 } Shape & Triangle & Square & Pentangle \\
\hline Concave & 1.836 & 1.477 & 2.136 \\
Convex & 1.789 & 1.639 & 1.440 \\
\hline
\end{tabular}

symmetry is present. The presence of reflective symmetry does not add any further facilitation to that of rotational symmetry alone (on the contrary, it seems that there is a slight advantage for the situations with only rotational symmetry).

The significance of the interaction between concave versus convex shapes and number of sides seems to be due to a small improvement as number of sides increases in convex but not in concave shapes (see Table 3 ). This seems to be a residual effect of number of sides, as observed in Experiment 2. However, because of the narrow range of variation of this factor, this was not observed here. It also suggests that a greater number of sides can be useful for the system only when the shape has a certain compactness. This seems to be the cause of the significance of the threeway interaction, as does the fact that the values reported for the nonsymmetrical concave shapes were higher than those reported for the nonsymmetrical convex shapes, which suggests that convexity could have a facilitating influence even if the shape is not symmetrical.

\section{Discussion}

The fact that the analysis on the prototypes was not significant seems to be in contrast with the results of the previous experiment, in which regular polygons were also used, and where the effect of order of symmetry (coinciding with the number of sides) was significant. However, it must be noted that, in the present experiment, the number of sides of the prototypes was, respectively, 3 , 4 , and 5 , whereas in the previous experiment it ranged from 3 to 10 . It can therefore be concluded that, in the present experiment, the number of sides did not vary on a wide enough range to produce an effect (the effect of the number-of-sides factor was also not significant in the analysis on the experimental stimuli), as it did in the previous experiment.

In the analysis on the experimental stimuli, two of the three main effects were significant-concave versus convex shapes and symmetry type-while number of sides was not. 
The most interesting result is the significance of symmetry type. In fact, for the first time, it was demonstrated that symmetry has a role in facilitating center determination. Still more interesting is the finding that, while it is significantly more difficult to locate the center of a nonsymmetrical shape than that of a symmetrical one, there is no difference in locating the center of a shape with both kinds of symmetry or with rotational symmetry alone. This is, in some measure, counterintuitive. In fact, it would intuitively be expected that the presence of symmetry axes would facilitate the process of finding a shape's center. This renders the hypothesis made at the end of Experiment 1 (the symmetry axes intersection) unlikely.

The fact that rotational symmetry alone is as effective as both symmetries together suggests another hypothesis (which, at present, is only tentative): It is possible that, in looking for the center of a shape, the visual system takes a shortcut, such as working on a simpler approximation of the shape, in which it tries to avoid the small peripheral parts. If an approximation of the shape is created, a symmetrical shape would be easier to be approximated because, by applying the same approximation in every direction, the same amount of area will also be evaluated in each direction, leaving the location of the perceived center unaltered. This is particularly true of rotationally symmetrical shapes, which are slightly more compact and tend to become circular as their number of sides increases (although if reflective symmetry is equal to two or more, rotational symmetry is also present, even though it is possible for a shape with one axis of reflection to be oblong). It is therefore possible that, in finding the center, the visual system exploits rotational symmetry to render the task easier, while it does not make a similar use of reflective symmetry. (In fact, also in Experiment 1, the set with a much higher systematic error-Set 1-was the only one with only reflective symmetry.)

As for the concave versus convex factor, it can be observed that the main differences between concave and convex shapes are that concave shapes have inflections and thin apexes, whereas in the convex shapes of this experiment, these subtle elongations are not present and, of course, they have no inflections (see Figure 5). The idea suggested for explaining the symmetry effect seems to be applicable here as well. The process of approximating a concave shape would yield some imprecisions in the result, which are avoided when the figure has no protrusions and their removal is therefore not needed, as in the case of convex shapes.

The suggestion of a procedure of approximation to the shape in order to find its center seems plausible because it would not require the visual system to use different strategies for different shapes, and because it can apply to every kind of shape (even without symmetry), which was impossible for the previous hypothesis (the symmetry axes intersection). However, at this point, this hypothesis is highly undetermined. The main question is: which sort of approximation is actually used by the perceiver?
Another question to be answered is: which are the boundaries of the set of shapes to which such an approximation would apply?

Although it is not completely clear what strategy is used for finding the center, it can be concluded that symmetry provides valuable aid in this task, and that rotational symmetry alone is sufficient.

\section{GENERAL DISCUSSION}

If a shape is symmetrical, the visual system uses this structural property to locate the shape's center. To have this effect, the presence of rotational symmetry is sufficient; in fact, if reflective symmetry is also present, there is no further improvement.

The question of which mathematical center is closest to the perceptual center has been posed in the literature. In some cases, it proved to be the contour barycenter (Beghi et al., 1983), and in others (as well as in the four shapes with noncoinciding mathematical centers of Experiment 3), it was the vertices' barycenter (Davi, 1989). Davi, however, proposed the possibility that the perceptual center would follow a law of its own and that no mathematical center could properly describe it in all cases. The present work, in which general factors that are applicable to all the shapes were analyzed, is a step in this direction. Now it is known that presentation plane does not influence determination of the perceptual center, whereas determination is influenced by the number of sides of a figure (if this factor varies on a wide range) and by whether or not it is symmetrical. A law describing how the center of flat figures is perceptually determined should account for these facts.

\section{REFERENCES}

Beghi, L., Vicario, G. \& Zanforun, M. (1983). The perceptual centre of visual configurations. Atti e Memorie della Accademia Pasavina di Scienze, Lettere ed Arti: Parte III. Classe di Scienze Morali, Lettere ed Arti, 95, 133-148.

Corballis, M. C., Roldan, C. E. (1975). Detection of symmetry as a function of angular orientation. Journal of Experimental Psychology: Human Perception \& Performance, 1, 221-230.

DAvi, M. (1989). Il centro percettivo di figure piane. Giomale Italiano di Psicologia, 16, 45-60.

GArner, W. R., Clement, D. E. (1963). Goodness of pattern and pattern uncertainty. Joumal of Verbal Learning \& Verbal Behavior, 2, 446-452.

GATtI, A. (1924). Sull'apprezzamento del centro in alcune figure piane geometriche. Archivio ltaliano di Psicologia, 3, 227-244.

HARTLEY, A. A. (1982). The roles of axes of symmetry in orientation illusions. Perception \& Psychophysics, 31, 367-375.

HumphreYs, G. W. (1983). Reference frames and shape perception. Cognitive Psychology, 15, 151-196.

LOCHER, P. J., Nodine, C. F. (1973). Influence of stimulus symmetry on visual scanning patterns. Perception \& Psychophysics, 13. 408-412.

Olson, R. K., AtTNEAve, F. (1970). What variables produce similarity groupings? American Journal of Psychology, 83, 1-21.

PALMER, S. E. (1985). The role of symmetry in shape perception. Acta Psychologica, 59, 67-90.

Palmer, S. E., Hemenway, K. (1978). Orientation and symmetry: 
Effects of multiple, rotational, and near symmetries. Journal of Experimental Psychology: Human Perception \& Performance, 4, 691-702.

Proffitt, D. R., \& O'Brien, R. G. (1982). Standardizing distances of observations within shapes. Perception \& Psychophysics, 31, 302-305.

Proffit, D. R., Simmons, R. W. (1980). A revolving $x$-y plotter for studying the perception of rotational motions. Behavior Research Methods \& Instrumentation, 12, 601-604.

Proffitt, D. R., Thomas, M. A., \& O'Brien, R. G. (1983). The roles of contour and luminance distribution in determining perceived centers within shapes. Perception \& Psychophysics, 33, 63-71.

Rock, I. (1973). Orientation and form. New York: Academic Press.

Rock, I., \& Leaman, R. (1963). An experimental analysis of visual symmetry. Acta Psychologica, 21, 171-183.

SChulze, H. H., Baurichter, W., Geruing, W., \& Grobe, R. (1977). Independent feature processing in the visual system: The effects of symmetry and distance of components. Psychological Research, 39, 249-259.

ZUSNE, L. (1965). Moments of area and of the perimeter of visual form as predictors of discrimination performance. Journal of Experimental Psychology, 69, 213-220.
ZUSNE, L. (1970). Visual perception of form. New York: Academic Press.

\section{NOTE}

1. The hypothesized possibility that motion might facilitate center determination was motivated by the notion that perceived centers might "balance" rotations, much as objects can be balanced by the placement of a support under their centers of gravity. That is, if an object is viewed revolving about some point other than its perceived center, it appears to jiggle. Nullification of this jiggle should occur when the shape's center coincides with the origin of its rotation. This experiment did not, however, provide a strong test of this hypothesis. Among other factors, rotation speed may be important, and certainly the observer's task-getting the shape to move to a desired location-was quite different across the static and moving conditions.

(Manuscript received December 26, 1990; revision accepted for publication January $31,1992$. 